\title{
Proprotein Convertase Subtilisin/Kexin Type 7
}

National Cancer Institute

\section{Source}

National Cancer Institute. Proprotein Convertase Subtilisin/Kexin Type 7. NCI Thesaurus. Code C97658.

Proprotein convertase subtilisin/kexin type 7 (785 aa, $\sim 86 \mathrm{kDa}$ ) is encoded by the human PCSK7 gene. This protein is involved in the proteolytic processing of immature proteins. 